\section{Robust Step-Like Identification of Low-Order Process Model Under Nonzero Initial Conditions and Disturbance}

\author{
Tao Liu and Furong Gao
}

\begin{abstract}
Nonsteady initial process states, measurement noise and unexpected load disturbance are practical difficulties associated with model identification from step response tests. A robust identification method is proposed to overcome these practical problems. The proposed step-like test differs from the conventional step test in that not only the process transient response to the step change, but also the subsequent transient response from removing the step change, are used for model identification. Based on a general low-order model structure, linear regression equations are established through multiple integrals for parameter estimation. The influence of nonzero initial process states and load disturbance is specifically considered in such a linear regression equation. A feasible instrumental variable (IV) method is also given with strict proof for consistent estimation against measurement noise. Illustrative examples from the recent literature are performed to show the effectiveness and merits of the proposed identification method.
\end{abstract}

Index Terms-Instrumental variable (IV), least-squares (LS) fitting, nonzero initial conditions, step response identification.

\section{INTRODUCTION}

Step response identification has been widely practiced in process industry to obtain continuous-time parametric model for control system design and tuning [1]-[3]. Most of existing references have been developed under zero initial conditions or steady-state of the process. Many, e.g., [4]-[6], were based on fitting several representative points in the process transient response to a step change. Others, such as [7]-[10], presented least-squares (LS) fitting algorithms to enhance identification accuracy and robustness in the presence of measurement noise. The recent paper [11] suggested a frequency-sampling filter to facilitate identification efficiency from step tests.

Step response tests for model identification are usually performed after the process moves into the steady-state, i.e., the process operational level. Due to measurement noise or unexpected load disturbance, it is often difficult to know if such a steady state has reached for step tests that are suitable for the aforementioned identification methods. Besides, waiting for such 'steady' state to have a step test can be quite troublesome for those processes with slow time constant or long time delay. Recently, Ahmed et al. [12], [13] proposed two algorithms, one using an iterative procedure and the other based on LS fitting, by regarding the initial state of the process output and its derivatives as part of the parameters to be identified while assuming no load disturbance; Wang et al. [14] developed a relay-based identification method to cope with static load disturbance and nonzero initial process output, the idea of which was further extended in the recent paper [15]; Using pulse response tests, Hwang and Lai [16] proposed a so-called two-stage LS algorithm to accomodate for nonzero initial state of the process output.

Manuscript received April 10, 2008; revised August 24, 2008, September 18, 2008, and September 22, 2008. Current version published December 10, 2008. This work was supported in part by the Hong Kong Research Grants Council under Project 613107. Recommended by Associate Editor J.-F. Zhang.

The authors are with the Department of Chemical and Biomolecular Engineering, Hong Kong University of Science and Technology, Kowloon, Hong Kong (e-mail: liurouter@ieee.org; kefgao@ust.hk).

Color versions of one or more of the figures in this technical note are available online at http://ieeexplore.ieee.org.

Digital Object Identifier 10.1109/TAC.2008.2007172

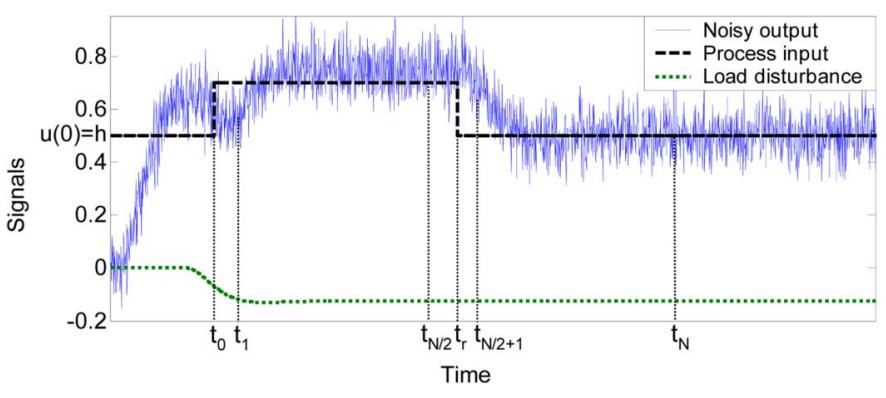

Fig. 1. Depiction of the proposed step-like test under nonzero initial conditions, measurement noise and load disturbance.

Note that with nonzero initial state of the process output, the corresponding process input is usually nonzero. Zero process input or its deviation from the steady-state value, however, had been used to develop identification algorithms in the recent step identification papers [12], [13] and non-step [14]-[16]. Besides, static load disturbance is only a specific type of load disturbances that are likely to occur in engineering test(s) for identification. Using directly the raw step response data, a robust identification method is proposed here that allows for nonzero initial process conditions and nonstatic load disturbance. Low-order model structure, first-order-plus-dead-time (FOPDT) or second-orderplus-dead-time (SOPDT) with or without right-half-plane (RHP) zero, is considered for identification, in view of that such models are most widely used for controller tuning in engineering practice. The proposed step-like test differs from the conventional step test in that the process transient response from removing the step change is also recorded for identification. As illustrated in Fig. 1, where $t_{0}$ denotes the time for adding the step change, the time for subsequently removing the step change, denoted as $t_{r}$, is determined by the user in terms of that the transient process response to adding the step change has nearly completed. Therefore, the proposed test also differs from the conventional pulse or rectangular wave test where the duration of the excitation signal is predetermined. By developing the corresponding identification algorithm through multiple integrals, we show that a conventional step test is not suitable for identification under nonzero initial process conditions and load disturbance.

\section{IDENTIFICATION METHOD}

1) Proposed Step-Like Test: As well known from existing step response identification methods, only the process output data of transient response to a step change are useful for model identification. To effectively pick up such data from adding the step change and subsequently removing it in the proposed test, the step change should be added to stir up observable dynamic response, and then be removed when observing that the process response has nearly entered into a steady state, as illustrated in Fig. 1. Note that both segments of the step response data, one for adding the step change (i.e., $t_{1} \sim t_{N / 2}$ ) and the other for removing it (i.e., $t_{N / 2+1} \sim t_{N}$ ), are used herein. An important merit of such choice is that the excitation sequence and the corresponding output sequence composed of the two segments data are no longer correlated to the time sequence. The incorrelate relationship will be nicely used in the identification algorithms developed later.

When adding the step change, it is suggested to monitor the process output trend. If the initial output, $y\left(t_{0}\right)$, has a trend of decreasing, i.e., $\dot{y}\left(t_{0}\right)<0$, a positive step change to the process input $(u)$ is suggested, so that an obvious turning point in the output response can be observed, 
which may be referenced to take the starting point $\left(t_{1}\right)$ of the first segment of step response data for identification. If the initial output has a trend of increasing, a negative step change should be used accordingly. If the trend of initial output cannot be observed clearly, particularly in the presence of measurement noise as shown in Fig. 1, either a positive or a negative step change may be used, the magnitude of which should be set reasonably large to yield an admissible output fluctuation. The last point $\left(t_{N / 2}\right)$ of the first segment data may be taken as or slightly earlier than the time for removing the step change, where $N$ is the total number of step response data used for identification.

For removing the step change, it is ideal to do so after the output response to the step change has completely entered into a steady state. However, it may be difficult to have such an observation in the presence of measurement noise and load disturbance. It is therefore suggested to remove the step change when observing that the output response has almost or nearly entered into a steady state. Correspondingly, the obvious turning point of the output response from removing the step change may be referenced to take the starting point $\left(t_{N / 2+1}\right)$ of the second segment data. The last point $\left(t_{N}\right)$ of the second segment data may be taken roughly after the output response has almost recovered to a steady state.

To have a good trade-off between computation efficiency and identification accuracy, $N$ is suggested as $50-200$.

2) Identification Algorithms: For identification of a low-order process model, a representative model structure is

$$
G=\frac{b_{1} s+b_{0}}{a_{2} s^{2}+a_{1} s+1} e^{-\theta s}
$$

which corresponds to the time domain response of

$$
a_{2} \ddot{y}(t)+a_{1} \dot{y}(t)+y(t)=b_{1} \dot{u}(t-\theta)+b_{0} u(t-\theta)+l(t)
$$

where $y(t)$ denotes the process output, $u(t)$ the process input, and $l(t)$ the deviation of the process output arising from load disturbance. $\theta$ denotes the process time delay, and $b_{0}$ is customarily named as the process gain. For $b_{1}<0$, (1) or (2) indicates a SOPDT model with a RHP zero. $b_{1}=0$ corresponds to a SOPDT model with three response types, underdamped, critically damped and overdamped. $b_{1}=0$ and $a_{2}=0$ lead to a FOPDT model.

For clarity, the following development of identification algorithms is detailed for obtaining a SOPDT model with a zero, and then is briefly summarized for obtaining a SOPDT model without zero or a FOPDT model.

\section{A. SOPDT Model With a Zero}

Given nonzero initial conditions of $y\left(t_{0}\right) \neq 0$ and $u\left(t_{0}\right)=h, h \in$ $\mathbb{R}$, by using a time shift of $t_{0}$, i.e., letting $t_{0}=0$, it follows for the proposed step-like test that

$$
u(t)= \begin{cases}h, & t \leq 0 \\ h+\Delta h, & 0<t \leq \tilde{t}_{r} \\ h, & t>\tilde{t}_{r}\end{cases}
$$

where $\tilde{t}_{r}=t_{r}-t_{0}$. To facilitate developing the identification algorithm, we may reformulate (2) as

$$
a_{2} \ddot{y}(t)+a_{1} \dot{y}(t)+y(t)=b_{1} \dot{u}(t)+b_{0} u(t)+l(t)
$$

with a time shifted description of

$$
u(t)= \begin{cases}h, & 0 \leq t \leq \theta \\ h+\Delta h, & \theta<t \leq \tilde{t}_{r}+\theta \\ h, & t>\tilde{t}_{r}+\theta\end{cases}
$$

Hence, for $t_{1} \leq t \leq t_{r}+\theta$, where $t_{1}>t_{0}+\theta$, it can be derived in terms of $t_{0}=0$ and (5) that

$$
\begin{aligned}
\int_{0}^{t} u(t) d t & =\int_{0}^{\theta} h d t+\int_{\theta}^{t}(h+\Delta h) d t \\
& =(h+\Delta h) t-\Delta h \theta \\
\int_{0}^{t} \dot{u}(t) d t & =\int_{0}^{\theta_{-}} 0 d t+\int_{\theta_{-}}^{\theta_{+}} \dot{u}(t) d t+\int_{\theta_{+}}^{t} 0 d t=\Delta h .
\end{aligned}
$$

Similarly, it can be derived for $t_{r}+\theta<t \leq t_{N}$ that

$$
\begin{aligned}
\int_{0}^{t} u(t) d t= & \int_{0}^{\theta} h d t+\int_{\theta}^{\tilde{t}_{r}+\theta}(h+\Delta h) d t+\int_{\tilde{t}_{r}+\theta}^{t} h d t \\
= & \Delta h \tilde{t}_{r}+h t \\
\int_{0}^{t} \dot{u}(t) d t= & \int_{0}^{\theta}-0 d t+\int_{\theta_{-}}^{\theta} \dot{u}(t) d t+\int_{\theta_{+}}^{\tilde{t}_{r}+\theta_{-}} 0 d t \\
& +\int_{\tilde{t}_{r}+\theta_{-}}^{\tilde{t}_{r}+\theta_{+}} \dot{u}(t) d t+\int_{\tilde{t}_{r}+\theta_{+}}^{t} 0 d t \\
= & 0 .
\end{aligned}
$$

Denote multiple integrals for a time function of $f(t)$ as

$$
\begin{aligned}
\int_{[0, t]}^{(m)} f(t) & =\int_{0}^{t} \int_{0}^{\tau_{m-1}} \ldots \int_{0}^{\tau_{1}} f\left(\tau_{0}\right) d \tau_{0} d \tau_{1} \ldots d \tau_{m-1}, \\
m & \geq 1 .
\end{aligned}
$$

For $t_{1} \leq t \leq t_{r}+\theta$, by using (6) it follows that:

$$
\begin{aligned}
\int_{[0, t]}^{(2)} u(t) & =\int_{0}^{\theta} \int_{0}^{\tau_{1}} h d \tau_{0} d \tau_{1}+\int_{\theta}^{t}\left[(h+\Delta h) \tau_{1}-\Delta h \theta\right] d \tau_{1} \\
& =\frac{h+\Delta h}{2} t^{2}-\Delta h t \theta+\frac{\Delta h}{2} \theta^{2} .
\end{aligned}
$$

Analogously, it can be derived as (12)-(17), shown at the bottom of the next page.

By triply integrating both sides of (4) and rearranging the resulting equation using (13), (15), (16) and (17), we obtain the LS form of parameter estimation

$$
\psi(t)=\phi^{T}(t) \gamma+\varepsilon(t)
$$

where $\varepsilon(t)$ denotes the residual error, and (19)-(23), shown at the bottom of the next page.

Note that in the parameter vector $\gamma$ for estimation, $\eta_{i}(i=$ $0,1,2, \ldots, q)$ are used for Maclaurin series approximation of the responses resulting from nonzero initial states of the process output, e.g., $y(0)$ and $\dot{y}(0)$ in (16) and (17), and load disturbance, according to the linear superposition principle for analysis of system response. The effects of $y(0), \dot{y}(0)$ and $l(t)$ to the LS fitting of (18) are approximately counted as

$$
\hat{Q}(t)=\sum_{j=0}^{q} \eta_{j} t^{j}
$$


which is based on the fact that the time domain response arising from nonzero initial process conditions and load disturbance can be generally expressed in the form of

$$
Q(t)=\sum_{j=0}^{m} \alpha_{j} e^{-\beta_{j} t}
$$

where $\alpha_{j}=\sum_{i=0}^{r} c_{i} t^{i}, c_{i} \in \mathbb{R}, \operatorname{Re}\left(\beta_{j}\right) \in \mathbb{R}_{+}, r$ is limited by the number of repetitive poles of the process and the input type, $m$ is the number of different poles of the process, and there exist

$$
\begin{aligned}
e^{-\operatorname{Re}\left(\beta_{j}\right) t}= & 1-\operatorname{Re}\left(\beta_{j}\right) t+\frac{\left[\operatorname{Re}\left(\beta_{j}\right)\right]^{2}}{2 !} t^{2}+\frac{\left[-\operatorname{Re}\left(\beta_{j}\right)\right]^{3}}{3 !} t^{3} \\
& +\cdots+\frac{\left[-\operatorname{Re}\left(\beta_{j}\right)\right]^{n}}{n !} t^{n}+R_{n}(t) \\
\lim _{t \rightarrow \infty} R_{n}(t)= & \lim _{t \rightarrow \infty} \frac{\left[-\operatorname{Re}\left(\beta_{j}\right) t\right]^{n+1}}{(n+1) !} e^{-\delta \operatorname{Re}\left(\beta_{j}\right) t}=0 \\
\delta \in & \in(0,1)
\end{aligned}
$$

For instance, if there exists no load disturbance, i.e., $l(t)=0$, it can be concluded using (16) and (17) that $q=2$ in (24) is sufficient to solve the LS fitting of (18). For the presence of a static load disturbance, i.e., $l(t)=c, c \in \mathbb{R}$, it can be similarly verified that $q=3$ is sufficient for parameter estimation if the model structure matches the process.

For the case that a nonstatic load disturbance occurs in the proposed test, the order of $q$ depends on the achievable approximation of (24) for representing the corresponding response with respect to the time used in the test. This may be used to explain why it is necessary to remove the step change after the output response has almost entered into a steady state. Note that the influence from a time varying disturbance that cannot be effectively approximated by (24) may confuse the LS fitting of (18). In such a case, the proposed test may be simply repeated for verification, owing to that the proposed algorithms are given for application with nonzero initial process states.

To determine $q$ for solving the LS fitting of (18), a statistical hypothesis testing may be used. That is, with an initial estimation of $q=3$ for iteration in terms of the same transient response data collected for identification, an updating law of $q(k+1)=q(k)+1$ may be used if

$$
\begin{aligned}
\int_{[0, t]}^{(2)} u(t) & = \begin{cases}\frac{h+\Delta h}{2} t^{2}-\Delta h t \theta+\frac{\Delta h}{2} \theta^{2} & \theta<t \leq \tilde{t}_{r}+\theta \\
\frac{h}{2} t^{2}+\Delta h \tilde{t}_{r} t-\frac{\Delta h}{2} \tilde{t}_{r}^{2}-\Delta h \tilde{t}_{r} \theta & t>\tilde{t}_{r}+\theta .\end{cases} \\
\int_{[0, t]}^{(3)} u(t) & = \begin{cases}\frac{h+\Delta h}{6} t^{3}-\frac{\Delta h}{2} t^{2} \theta+\frac{\Delta h}{2} t \theta^{2}-\frac{\Delta h}{6} \theta^{3}, & \theta<t \leq \tilde{t}_{r}+\theta \\
\frac{h}{6} t^{3}+\frac{\Delta h}{2} \tilde{t}_{r} t^{2}-\frac{\Delta h}{2} \tilde{t}_{r}^{2} t+\frac{\Delta h}{6} \tilde{t}_{r}^{3}+\left(\frac{\Delta h}{2} \tilde{t}_{r}^{2}-\Delta h \tilde{t}_{r} t\right) \theta+\frac{\Delta h}{2} \tilde{t}_{r} \theta^{2}, & t>\tilde{t}_{r}+\theta .\end{cases} \\
\int_{[0, t]}^{(2)} \dot{u}(t) & = \begin{cases}\Delta h(t-\theta), & \theta<t \leq \tilde{t}_{r}+\theta \\
\Delta h \tilde{t}_{r}, & t>\tilde{t}_{r}+\theta .\end{cases} \\
\int_{[0, t]}^{(3)} \dot{u}(t) & = \begin{cases}\frac{\Delta h}{2} t^{2}-\Delta h t \theta+\frac{\Delta h}{2} \theta^{2}, & \theta<t \leq \tilde{t}_{r}+\theta \\
\Delta h \tilde{t}_{r} t-\frac{\Delta h}{2} \tilde{t}_{r}^{2}-\Delta h \tilde{t}_{r} \theta & t>\tilde{t}_{r}+\theta .\end{cases} \\
\int_{[0, t]}^{(3)} \dot{y}(t) & =\int_{[0, t]}^{(2)} y(t)-\frac{1}{2} y(0) t^{2} \\
\int_{[0, t]}^{(3)} \ddot{y}(t) & =\int_{[0, t]}^{(1)} y(t)-y(0) t-\frac{1}{2} \dot{y}(0) t^{2}
\end{aligned}
$$

$$
\begin{aligned}
& \left\{\begin{aligned}
\psi(t) & =\int_{[0, t]}^{(3)} y(t), \\
\phi(t) & =\left[-\int_{[0, t]}^{(1)} y(t),-\int_{[0, t]}^{(2)} y(t), F_{0}(t), F_{1}(t), F_{2}(t), F_{3}(t), 1, t, t^{2}, \ldots, t^{q}\right]^{T} \\
\gamma & =\left[a_{2}, a_{1}, b_{0}, b_{0} \theta-b_{1}, b_{0} \theta^{2}-2 b_{1} \theta, b_{0} \theta^{3}-3 b_{1} \theta^{2}, \eta_{0}, \eta_{1}, \eta_{2}, \ldots, \eta_{q}\right]^{T} .
\end{aligned}\right. \\
& F_{0}(t)= \begin{cases}\frac{h+\Delta h}{6} t^{3}, & \theta<t \leq \tilde{t}_{r}+\theta \\
\frac{h}{6} t^{3}+\frac{\Delta h}{2} \tilde{t}_{r} t^{2}-\frac{\Delta h}{2} \tilde{t}_{r}^{2} t+\frac{\Delta h}{6} \tilde{t}_{r}^{3}, & t>\tilde{t}_{r}+\theta .\end{cases} \\
& F_{1}(t)= \begin{cases}-\frac{\Delta h}{2} t^{2}, & \theta<t \leq \tilde{t}_{r}+\theta \\
\frac{\Delta h}{2} \tilde{t}_{r}^{2}-\Delta h \tilde{t}_{r} t, & t>\tilde{t}_{r}+\theta .\end{cases} \\
& F_{2}(t)= \begin{cases}\frac{\Delta h}{2} t & \theta<t \leq \tilde{t}_{r}+\theta \\
\frac{\Delta h}{2} \tilde{t}_{r} & t>\tilde{t}_{r}+\theta .\end{cases} \\
& F_{3}(t)= \begin{cases}-\frac{\Delta h}{6}, & \theta<t \leq \tilde{t}_{r}+\theta \\
0, & t>\tilde{t}_{r}+\theta .\end{cases}
\end{aligned}
$$


the following convergent condition is not satisfied at the $k$ th iteration step

$$
\mathrm{ERR}=\sqrt{\frac{1}{p} \sum_{i=1}^{p}\left[\gamma^{[k]}(i)-\gamma^{[k-1]}(i)\right]^{2}}<\sigma
$$

where $\sigma$ is a user-specified threshold for assessing the fitting standard deviation of the model parameters, and $p$ is the number of the model parameters to be identified.

Hence, by using the proposed two segments data and letting $\Psi=$ $\left[\psi\left(t_{1}\right), \psi\left(t_{2}\right), \ldots, \psi\left(t_{N}\right)\right]^{T}$ and $\Phi=\left[\phi\left(t_{1}\right), \phi\left(t_{2}\right), \ldots, \phi\left(t_{N}\right)\right]^{T}$, we can establish a LS algorithm for parameter estimation

$$
\Psi=\Phi \gamma
$$

Accordingly, the parameter vector can be solved as

$$
\gamma=\left(\Phi^{T} \Phi\right)^{-1} \Phi^{T} \Psi
$$

To clarify the invertibility of $\Phi^{T} \Phi$ for computation, we give

Proposition 1: $\Phi^{T} \Phi$ is guaranteed invertible if using the proposed step-like test.

Proof: Note that the last $q$ columns in $\Phi$ are all time vectors with different orders, so they are linearly independent of each other. The first two columns in $\Phi$, sequences of single and double integrals for the process output, are obviously linearly independent of the last $q$ columns. Owing to the proposed test, $F_{j}(t)$ for $j=0,1,2,3$ are all piecewise continuous-time functions, so the corresponding columns in $\Phi$ are not only linearly independent of each other, but also linearly independent of the other columns in $\Phi$. Therefore, $\Phi$ is guaranteed as full column rank. It follows from $\operatorname{rank}\left(\Phi^{T} \Phi\right)=\operatorname{rank}(\Phi)$ that $\Phi^{T} \Phi$ is invertible.

Remark 1: If a conventional step test is used for identification, the transient response data correspond only to the first segment data of the proposed test. It can be seen from (20)-(23) that the resulting $F_{j}(t)$ for $j=0,1,2$ will all be time functions, while $F_{3}(t)$ becomes a constant. The corresponding columns will definitely be linearly dependent of the last $q+1$ columns in $\Phi$, causing $\Phi^{T} \Phi$ not invertible. It is thus demonstrated that a conventional step test is not suitable for parameter estimation with nonzero initial process conditions and load disturbance. $\diamond$

Remark 2: Unbiased estimation, i.e., $\varepsilon(t)=0$ in (18), can be attained thoroughly if the model structure matches the process, owing to the influence of nonzero initial conditions and load disturbance has been specifically considered as seen from (24)-(27). To accommodate for model mismatch as encountered in practice, a user-specified identification accuracy as shown in (28) is preferred for computation, unavoidably at the cost of response estimation error to some extent. $\diamond$

In the sequel, the model parameters can be retrieved from $\gamma(i)(i=$ $1,2, \ldots, p)$ as

$$
\begin{aligned}
{\left[a_{2}, a_{1}, b_{0}, b_{1}, \theta\right]^{T} } & =[\gamma(1), \gamma(2), \gamma(3) \\
& \left. \pm \sqrt{\gamma^{2}(4)-\gamma(3) \gamma(5)},\left[b_{1}+\gamma(4)\right] / \gamma(3)\right]^{T}
\end{aligned}
$$

Obviously, a positive $b_{1}$ indicates a minimum phase process, and a negative $b_{1}$ corresponds to an inverse response process.

It should be noted that unlike multiple independent fitting conditions as studied in the recent papers [17], [18], the redundant fitting condition, $\gamma(6)$, which is resulted from the triple integrals to (4), is parasitic to the independent fitting conditions of $\gamma(4)$ and $\gamma(5)$, and thus is useless to retrieve the model parameters.

In the presence of measurement noise, $\zeta(t)$, there exists $\hat{y}(t)=$ $y(t)+\zeta(t)$, where $\hat{y}(t)$ denotes the actually measured process output. Substituting it into (4) and then triply integrating both sides of (4), we can obtain

$$
\psi(t)=\phi^{T}(t) \gamma+\nu(t)
$$

where $\psi(t)=\int_{[0, t]}^{(3)} \hat{y}(t), \nu(t)=a_{2} \int_{[0, t]}^{(1)} \zeta(t)+a_{1} \int_{[0, t]}^{(2)} \zeta(t)+$ $\int_{[0, t]}^{(3)} \zeta(t), \quad \phi(t) \quad=\quad\left[-\int_{[0, t]}^{(1)} \hat{y}(t),-\int_{[0, t]}^{(2)} \hat{y}(t), F_{0}(t)\right.$, $\left.F_{1}(t), F_{2}(t), F_{3}(t), 1, t, t^{2}, \ldots, t^{q}\right]^{T}$, and $\gamma$ is the same as in (19).

It is seen from (32) that $\phi^{T}(t)$ is now correlated with $\nu(t)$. Thereby, parameter estimation from (30) may not be consistent according to the parameter estimation theory [3]. A feasible instrumental variable (IV) for consistent estimation is proposed in the following proposition:

Proposition 2: With a IV matrix chosen as $Z=\left[z_{1}, z_{2}, \ldots, z_{N}\right]^{T}$ where $z_{i}=\left[1 / t_{i}^{p+1}, 1 / t_{i}^{p}, \ldots, 1 / t_{i}, 1, t_{i}, t_{i}^{2}, \ldots, t_{i}^{q}\right]^{T}$, which satisfies the two limiting conditions: 1$)$. the inverse of $\lim _{N \rightarrow \infty}\left(Z^{T} \Phi\right) / N$ exists; 2). $\lim _{N \rightarrow \infty}\left(Z^{T} v\right) / N=0$, where $v=\left[\nu\left(t_{1}\right), \nu\left(t_{2}\right), \ldots, \nu\left(t_{N}\right)\right]^{T}$, a consistent parameter estimation is given by $\gamma=\left(Z^{T} \Phi\right)^{-1} Z^{T} \Psi$.

Proof: The two limiting conditions in Proposition 2 are sufficient for consistent estimation [3]. It is thus required to prove that the proposed IV matrix satisfies the two conditions.

For the first condition, which is equivalent to that $\left(Z^{T} \Phi\right) / N$ is guaranteed nonsingular for $N \rightarrow \infty$, we have shown that both $\Phi$ and $Z$ are full column rank for $N \geq \operatorname{dim}(\gamma)$. Note that

$$
\begin{aligned}
& \operatorname{rank}(Z)=\operatorname{rank}(\Phi) \\
& \operatorname{rank}(Z)=\operatorname{rank}\left(Z^{T}\right)=\operatorname{rank}\left(Z^{T} Z\right) \\
& \operatorname{rank}(\Phi)=\operatorname{rank}\left(\Phi^{T}\right)=\operatorname{rank}\left(\Phi^{T} \Phi\right) .
\end{aligned}
$$

Besides, there exists

$$
\left(\frac{1}{N} Z^{T} \Phi\right)^{T}\left(\frac{1}{N} Z^{T} \Phi\right)=\Phi^{T}\left(\frac{1}{N^{2}} Z Z^{T}\right) \Phi
$$

We may define here

$$
A=\frac{1}{N^{2}} Z Z^{T}
$$

For $0<t_{1}<t_{2}<\cdots<t_{N}$, it can be easily verified that

$$
X^{T} A X \geq 0, \quad \forall X \in \mathbb{R}^{N \times 1} \text {, and } X^{T} A X=0 \Leftrightarrow X=0
$$

which indicates that $A$ is positive definite. It follows that:

$$
\operatorname{rank}\left(\Phi^{T} A \Phi\right)=\operatorname{rank}(\Phi) .
$$

Therefore, we can conclude from (36) and (39) that $\left(Z^{T} \Phi\right) / N$ is nonsingular.

For the second condition, it follows for $N \rightarrow \infty$ that $\zeta\left(t_{i}\right)(i=$ $1,2, \ldots, N)$ may be viewed as a white noise sequence with zero mean. The corresponding $\nu\left(t_{i}\right)(i=1,2, \ldots, N)$ can thus be determined as a random distribution with zero mean, which is independent of the time origin or $t_{0}$ as used in the proposed test. Therefore, the random vector $v$ is uncorrelated with the rows of $Z^{T}$ that are time vectors with different orders, implying that the second condition is also satisfied.

\section{B. SOPDT Model Without Zero}

By letting $b_{1}=0$, the above identification algorithm can directly be applied to obtain a SOPDT model without zero. The only dif- 
ference is that the parameter vector in (19) should be rectified as $\gamma=\left[a_{2}, a_{1}, b_{0}, b_{0} \theta, b_{0} \theta^{2}, b_{0} \theta^{3}, \eta_{0}, \eta_{1}, \eta_{2}, \ldots, \eta_{q}\right]^{T}$. Accordingly, the model parameters can be retrieved as

$$
\left[a_{2}, a_{1}, b_{0}, \theta\right]^{T}=\left[\gamma(1), \gamma(2), \gamma(3), \frac{\gamma(4)}{\gamma(3)}\right]^{T} .
$$

\section{FOPDT Model}

By doubly integrating both sides of (4) and using (12) and (14), we can formulate a LS fitting similar to (18), for which

$$
\begin{aligned}
\langle(t) & =\int_{[0, t]}^{(2)} y(t), \\
\phi(t) & =\left[-\int_{[0, t]}^{(1)} y(t), F_{0}(t), F_{1}(t), F_{2}(t), 1, t, t^{2}, \ldots, t^{q}\right]^{T} \\
\gamma & =\left[a_{1}, b_{0}, b_{0} \theta, b_{0} \theta^{2}, \eta_{0}, \eta_{1}, \eta_{2}, \ldots, \eta_{q}\right]^{T} \\
F_{0}(t) & = \begin{cases}\frac{h+\Delta h}{2} t^{2}, & \theta<t \leq \tilde{t}_{r}+\theta \\
\frac{h}{2} t^{2}+\Delta h \tilde{t}_{r} t-\frac{\Delta h}{2} \tilde{t}_{r}^{2} & t>\tilde{t}_{r}+\theta\end{cases} \\
F_{1}(t) & = \begin{cases}-\Delta h t, & \theta<t \leq \tilde{t}_{r}+\theta \\
-\Delta h \tilde{t}_{r}, & t>\tilde{t}_{r}+\theta\end{cases} \\
F_{2}(t) & = \begin{cases}\frac{\Delta h}{2}, & \theta<t \leq \tilde{t}_{r}+\theta \\
0, & t>\tilde{t}_{r}+\theta\end{cases}
\end{aligned}
$$

It can be easily verified that $q=1$ is sufficient to solve the LS fitting if there exists no load disturbance, and $q=2$ is sufficient for the presence of a static load disturbance.

Accordingly, the model parameters can be derived using (30) and (41) as

$$
\left[a_{1}, b_{0}, \theta\right]^{T}=\left[\gamma(1), \gamma(2), \frac{\gamma(3)}{\gamma(2)}\right]^{T}
$$

For consistent estimation against measurement noise, the corresponding IV may be taken according to Proposition 2 as $z_{i}=\left[1 / t_{i}^{4}, 1 / t_{i}^{3}, 1 / t_{i}^{2}, 1 / t_{i}, 1, t_{i}, t_{i}^{2}, \ldots, t_{i}^{q}\right]^{T}$.

Remark 3: By letting $u(0)=0$ (i.e., $h=0$ for $t<t_{0}=0$ ), all the above identification algorithms can be directly applied for zero initial conditions, regardless of whether load disturbance exists or not. In such a case, $q=0$ may be used if there exists no load disturbance. Note that the proposed identification algorithms can be transparently extended to the case that the initial process input is also time varying, because its variation is usually under control in practice and thus can be explicitly known and expressed for computation.

\section{ILLUSTRATION}

In the following examples, the widely used criterion of transient response error to a unity step change [2], [3] is adopted to assess the achievable identification accuracy

$$
\operatorname{err}=\frac{1}{N_{s}} \sum_{k=1}^{N_{s}}\left[\widehat{y}\left(k T_{s}\right)-y\left(k T_{s}\right)\right]^{2}
$$

where $\widehat{y}\left(k T_{s}\right)$ and $y\left(k T_{s}\right)$ denotes the model and process responses, respectively, and $N_{s} T_{s}$ is the settling time.

\begin{tabular}{|c|c|c|c|}
\hline NSR & Example & Identified Model & err \\
\hline \multirow{4}{*}{0} & 1 & $1.2507 e^{-0.2536 s}$ & \multirow[t]{2}{*}{$3.88 \times 10^{-5}$} \\
\hline & & $\overline{0.2503 s^{2}+0.6994 s+1}$ & \\
\hline & \multirow[t]{2}{*}{2} & $(-4.000 s+1.0000) e^{-1.0187 s}$ & \multirow{2}{*}{$4.04 \times 10^{-6}$} \\
\hline & & $9.0000 s^{2}+2.4000 s+1$ & \\
\hline \multirow{4}{*}{$1 \%$} & 1 & $1.247 e^{-0.2501 s}$ & \multirow[t]{2}{*}{$6.19 \times 10^{-5}$} \\
\hline & \multirow{3}{*}{2} & $\overline{0.2548 s^{2}+0.7021 s+1}$ & \\
\hline & & $(-4.0039 \mathrm{~s}+0.9971) e^{-1.0189 s}$ & \multirow[t]{2}{*}{$2.76 \times 10^{-5}$} \\
\hline & & $9.0459 s^{2}+2.4125 s+1$ & \\
\hline \multirow{4}{*}{$10 \%$} & \multirow[t]{2}{*}{1} & $1.2202 e^{-0.2234 s}$ & \multirow{2}{*}{$9.22 \times 10^{-4}$} \\
\hline & & $\overline{0.2998 s^{2}+0.7153 s+1}$ & \\
\hline & \multirow[t]{2}{*}{2} & $(-4.0425 \mathrm{~s}+0.9726) e^{-0.9307 s}$ & \multirow{2}{*}{$1.08 \times 10^{-3}$} \\
\hline & & $9.421 s^{2}+2.5255 s+1$ & \\
\hline \multirow{4}{*}{$20 \%$} & \multirow[t]{2}{*}{1} & $1.1914 e^{-0.2338 s}$ & \multirow{2}{*}{$3.11 \times 10^{-3}$} \\
\hline & & $\overline{0.3209 s^{2}+0.6886 s+1}$ & \\
\hline & \multirow[t]{2}{*}{2} & $\underline{(-4.0968 s+0.9437) e^{-0.823 s}}$ & \multirow[t]{2}{*}{$4.4 \times 10^{-3}$} \\
\hline & & $9.8338 s^{2}+2.676 s+1$ & \\
\hline
\end{tabular}

Example 1: Consider the SOPDT process studied in the recent papers [15], [19], $G=1.25 e^{-0.234 s} /\left(0.25 s^{2}+0.7 s+1\right)$, i.e., $0.25 \ddot{y}(t)+$ $0.7 \dot{y}(t)+y(t)=1.25 u(t-0.234)+l(t)$.

Based on zero initial conditions and $l(t)=0$, Liu et al. [15] derived a SOPDT model using multiple step response tests, and Ahmed
TABLE I

IDENTIFICATION RESULtS AgAinst MEASUREMENT NOISE

et al. [19] developed a SOPDT algorithm using a random binary signal (RBS) for excitation. To illustrate the proposed method, assume that the initial conditions are $y\left(t_{0}\right)=0.6, \dot{y}\left(t_{0}\right)=-0.06$ and $u\left(t_{0}\right)=0.5$. In view of that the initial output has a trend of decreasing, a step change of $\Delta h=0.2$ is added at $t_{0}=3(s)$, ahead of which a load disturbance with a slow dynamics of $G_{d}=0.2 /(0.5 s+1)$ is injected into the process output at $t=2(s)$. The step change is removed at $t_{r}=10(s)$ before the load disturbance becomes steady. Using the output data in the time intervals of $[3.5,10](s)$ and $[10.5,20](s)$, the proposed method with $N=100$ and $q=8$ gives a model listed in Table I, indicating good accuracy. Note that the Maclaurin approximation for the effects from the nonzero initial states and the load disturbance converges at $q=8$ with a prescribed threshold of $\sigma=5 \%$.

To demonstrate identification robustness in the presence of measurement noise, assume that random noise of $N\left(0, \sigma_{N}^{2}=0.45 \%\right)$, causing the noise-to-signal ratio (NSR) to $N S R=10 \%$, is added to the output measurement, while a step disturbance with a magnitude of 0.1 is added to the process input at $t=2(\mathrm{~s})$, as shown in Fig. 1. Repeating the above test and using the output data in the time intervals of $[4,10](s)$ and $[11,25](s)$, which are obvious for observing the output dynamic responses to adding and removing the step change, the proposed method with $q=3$ gives a model listed in Table I, indicating good identification robustness. The results for $N S R=1 \%$ and $20 \%$ are also listed in Table I to show the achievable accuracy.

Example 2: Consider the SOPDT process with a RHP zero studied in refs.[8], [9], [13], [15], $G=(-4 s+1) e^{-s} /\left(9 s^{2}+2.4 s+1\right)$, i.e.,

$$
9 \ddot{y}(t)+2.4 \dot{y}(t)+y(t)=-4 \dot{u}(t-1)+u(t-1)+l(t) .
$$

Zero initial process conditions was assumed in [8], [9], [15], and nonzero initial conditions but with no load disturbance was assumed in [13]. For illustration, the initial conditions are herein assumed as $y(0)=0.5, \dot{y}(0)=-0.026$ and $u(0)=0.5$, which are picked up from the process transient response to a step input with a magnitude of 0.5 , while subject to a slowly changing load disturbance as in example 1. By performing a step-like test as in example 1 , the proposed method 


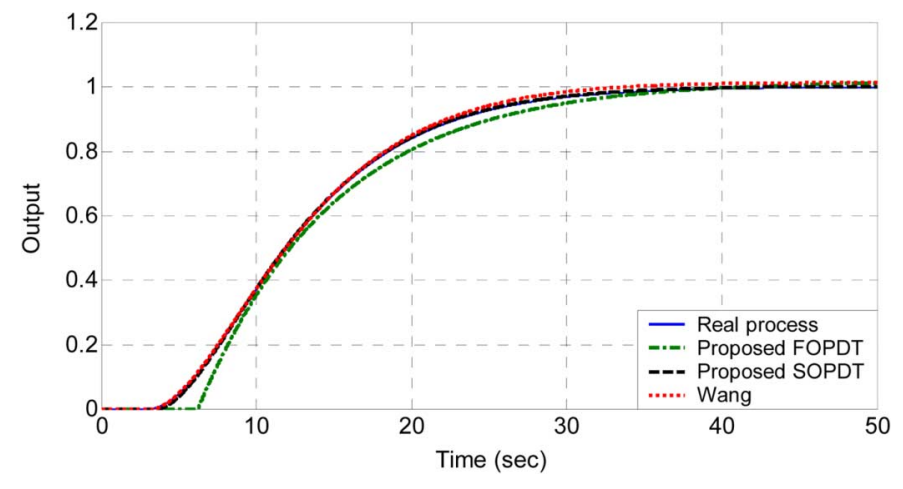

Fig. 2. Step response fitting for example 3.

with $N=100$ and $q=7$ in terms of the output response data in the time intervals of $[6.5,10](s)$ and $[15,35](s)$ gives a model listed in Table I, once again demonstrating good accuracy. It is also illustrated that using a longer time to collect the second segment data facilitates reducing the Maclaurin approximation order of $q$ in terms of $\sigma=5 \%$ as used in example 1. Repeating measurement noise tests as in example 1 and letting $t_{r}=35(\mathrm{~s})$, the corresponding results obtained using $q=3$ in terms of the output response data in the time intervals of $[8,35](\mathrm{s})$ and $[40,65](s)$ are also listed in Table I, again demonstrating good identification robustness.

Example 3: Consider the higher order process studied in the recent paper [14], $25 \ddot{y}(t)+35 \ddot{y}(t)+11 \dot{y}(t)+y(t)=u(t-2.5)+l(t)$, with the initial conditions of $y(0)=2, \dot{y}(0)=0.4, \ddot{y}(0)=-0.4$, and $u(0)=0$, together with a static load disturbance of $l(t)=1$. For illustration, the same initial conditions but with nonzero input of $u(0)=1.5$ are considered here. In view of that the initial output has a trend of increasing, a step change of $\Delta h=-0.5$ is added at $t_{0}=0(s)$ and then is removed at $t_{r}=10(s)$, the proposed method with $N=100$ and $q=3$ in terms of the output response data in the time intervals of $[5,10](s)$ and $[15,45](s)$ gives a FOPDT model, $G_{m}=1.0192 e^{-6.2227 s} /(8.8339 s+1)$, corresponding to err $=7.42 \times 10^{-4}$, and a SOPDT model, $G_{m}=1.0025 e^{-3.4681 s} /\left(25.2131 s^{2}+10.0171 s+1\right)$, corresponding to err $=1.32 \times 10^{-5}$. Wang et al. [14] derived a SOPDT model, $G_{m}=0.03411 e^{-3.11 s} /\left(s^{2}+0.3486 s+0.03366\right)$, corresponding to err $=1.46 \times 10^{-4}$. For comparison, the unity step responses of these models are shown in Fig. 2. It can be seen that both SOPDT models are capable of better fitting compared to the FOPDT model, while the proposed SOPDT model shows further improved fitting effect.

\section{CONCLUSION}

For general application of step response identification in the presence of nonsteady or even unknown initial process states, measurement noise and load disturbance, a robust step-like identification method has been proposed for obtaining widely used low-order process models of FOPDT and SOPDT with or without zero, which may also be transparently extended to obtain a specific higher order model. A distinguishing feature of the proposed step-like test is that the process transient response from removing the step change is also used for identification. It is therefore demonstrated that a conventional step test is not suitable for parameter estimation in the face of nonzero initial process conditions and load disturbance. With a user-specified threshold for convergence, the Maclaurin series approximation for representing the effects of nonzero initial conditions and load disturbance can be conveniently implemented in the proposed identification algorithms. Owing to that nonzero initial process conditions are considered, the proposed method can be easily repeated on line if unexpected load disturbance impedes convergence. The applications to three examples from the recent papers have demonstrated that the proposed identification method can achieve good accuracy.

\section{REFERENCES}

[1] D. E. Seborg, T. F. Edgar, and D. A. Mellichamp, Process Dynamics and Control, 2nd ed. Hoboken, NJ: Wiley, 2003.

[2] K. J. Åström and T. Hägglund, Advanced PID Control. Research Triangle Park, NC: ISA Society of America, 2005.

[3] L. Ljung, System Identification: Theory for the User, 2nd ed. Englewood Cliffs, NJ: Prentice Hall, 1999.

[4] H. Rake, "Step response and frequency response methods," Automatica, vol. 16, pp. 519-526, 1980.

[5] G. P. Rangaiah and P. R. Krishnaswamy, "Estimating second-order dead time parameters from underdamped process transients," Chem. Eng. Sci., vol. 51, pp. 1149-1155, 1996.

[6] H. P. Huang, M. W. Lee, and C. L. Chen, "A system of procedures for identification of simple models using transient step response," Ind. Eng. Chem. Res., vol. 40, pp. 1903-1915, 2001.

[7] Q. Bi, W. J. Cai, E. L. Lee, Q. G. Wang, C. C. Hang, and Y. Zhang, "Robust identification of first-order plus dead-time model from step response," Control Eng. Practice, vol. 7, pp. 71-77, 1999.

[8] Q. G. Wang and Y. Zhang, "Robust identification of continuous systems with dead-time from step responses," Automatica, vol. 37, pp. 377-390, 2001.

[9] Q. G. Wang, X. Guo, and Y.Zhang, "Direct identification of continuous time delay systems from step responses," J. Process Control, vol. 11, pp. 531-542, 2001.

[10] S. Y. Li, W. J. Cai, H. Mei, and Q. Xiong, "Robust decentralized parameter identification for two-input two-output process from closed-loop step responses," Control Eng. Practice, vol. 13, pp. 519-531, 2005.

[11] L. Wang and W. R. Cluett, "Frequency-sampling filters: An improved model structure for step-response identification," Automatica, vol. 33, pp. 939-944, 1997.

[12] S. Ahmed, B. Huang, and S. L. Shah, "Novel identification method from step response," Control Eng. Practice, vol. 15, pp. 545-556, 2007.

[13] S. Ahmed, B. Huang, and S. L. Shah, "Identification from step responses with transient initial conditions," J. Process Control, vol. 18, pp. 121-130, 2008.

[14] Q. G. Wang, M. Liu, C. C. Hang, and W. Tang, "Robust process identification from relay tests in the presence of nonzero initial conditions and disturbance," Ind. Eng. Chem. Res., vol. 45, pp. 4063-4070, 2006.

[15] M. Liu, Q. G. Wang, B. Huang, and C. C. Hang, "Improved identification of continuous-time delay processes from piecewise step tests," $J$. Process Control, vol. 17, pp. 51-57, 2007.

[16] S. H. Hwang and S. T. Lai, "Use of two-stage least-squares algorithms for identification of continuous systems with time delay based on pulse responses," Automatica, vol. 40, pp. 1561-1568, 2004.

[17] T. Liu and F. Gao, "Alternative identification algorithms for obtaining a first-order stable/unstable process model from a single relay feedback test," Ind. Eng. Chem. Res., vol. 47, pp. 1140-1149, 2008.

[18] T. Liu and F. Gao, "A systematic approach for on-line identification of second-order process model from relay feedback test," AIChE J., vol. 54 , pp. 1560-1578, 2008.

[19] S. Ahmed, B. Huang, and S. L. Shah, "Parameter and delay estimation of continuous-time models using a linear filter," J. Process Control, vol. 16, pp. 323-331, 2006. 